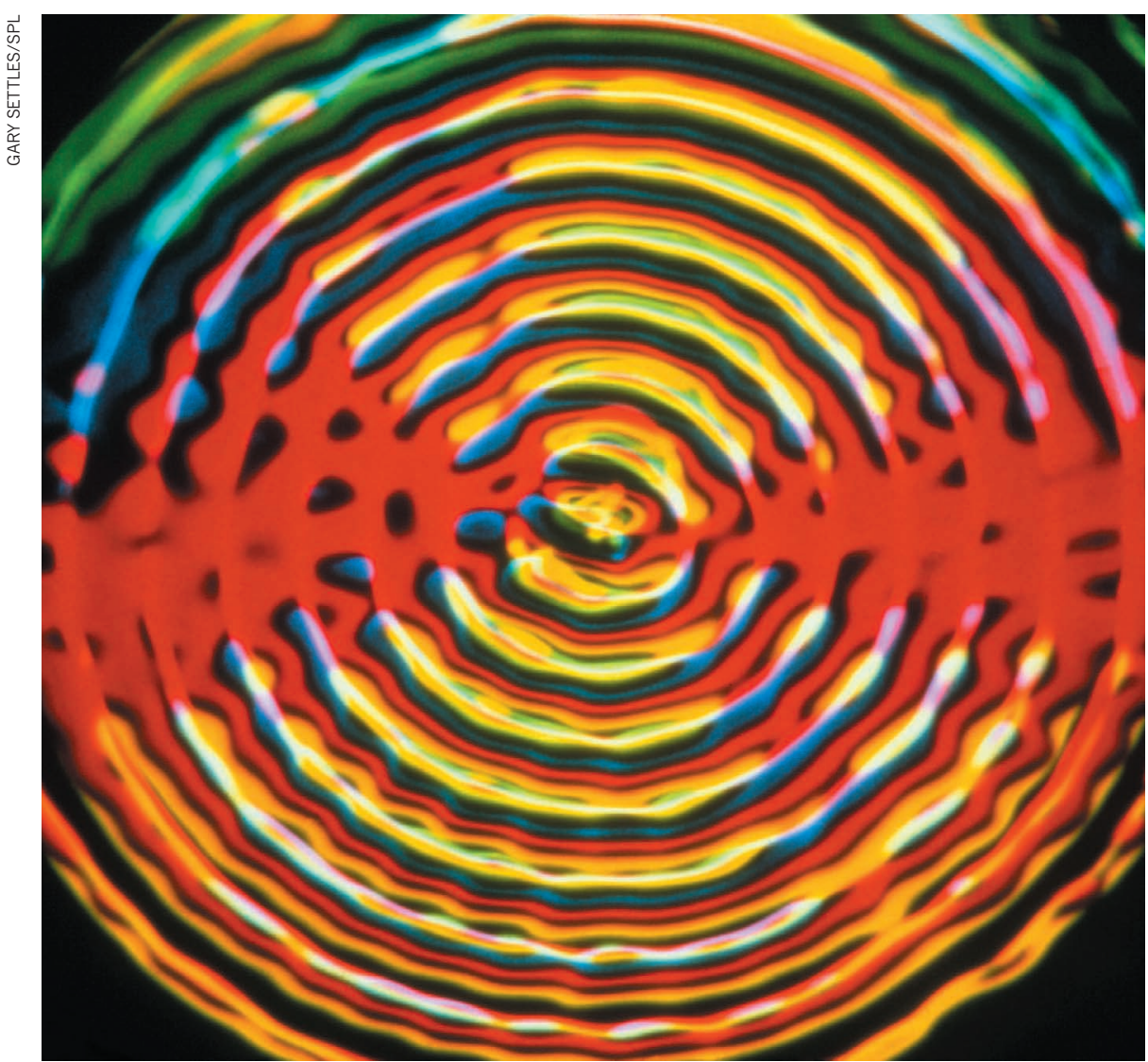

A Schlieren photograph — which shows density changes in fluids - of ripples in mercury.

\title{
PHYSICS
}

\section{A classical toolkit}

\section{Malcolm Longair extols a long-heralded tome by Roger Blandford and 2017 Nobel laureate Kip Thorne.}

$\mathrm{I}$ $\mathrm{t}$ is a matter for celebration when two illustrious theoreticians such as Kip Thorne and Roger Blandford provide an in-depth description of the fundamentals of classical physics. Modern Classical Physics is the fruit of three decades or more of teaching this material to first-year graduates. And it is informed by the duo's numerous major research contributions - Thorne, for example, won a share in this year's physics Nobel prize. The sheer amount of material covered and the effort that has gone into condensing it into a single, beautifully produced volume is extraordinarily impressive. The result is a book of some 1,550 pages, weighing in at around 3.5 kilograms.

Covering the six major subject areas listed in the subtitle - from optics to relativity - the book presupposes a strong understanding of the standard approaches to classical mechanics, electromagnetic theory, elementary thermodynamics and applied mathematics. Lagrangian and Hamiltonian mechanics and dynamics, as coordinateindependent descriptions of the physical laws, are assumed to be in the reader's armoury.

Basic quantum mechanics is also assumed. It comes as a surprise at first to find how ubiquitous Planck's constant $h$ - which always signifies the involvement of quantum concepts - is in several of their arguments on classical physics. There are, of course, splendid precedents for this. One is in classical statistical physics, which uses the concept of discrete energy levels, now deeply embedded in quantum mechanics, as a trick to calculate exactly energy distribution (the separation of these levels is eventually set equal to zero, to recover the classical picture). Thorne and Blandford use the same tactic, but with the quantum-energy elements $h v$, where $v$ is frequency, and set $h$ equal to zero to obtain the desired classical continuous result. The authors extend this approach successfully

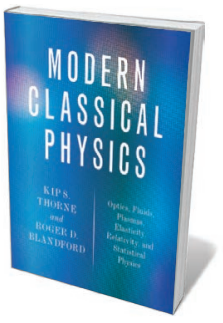

Modern Classical Physics: Optics, Fluids, Plasmas, Elasticity,

Relativity, and

Statistical Physics KIPS. THORNE \& ROGER D. BLANDFORD Princeton University Press: 2017.

ciati ciating its content, and (although they do not like being told this) students have to find their own way of understanding it. A single lecture course on any of the topics here is not enough to give the deep insights needed to make creative use of the tools of the trade.

Thorne and Blandford take every opportunity to help the reader and emphasize the coherence and long-range connections between apparently different areas of study. And the book is contemporary in content and feel: the collapse of the World Trade Center's twin towers on 11 September 2001, the first detection of gravitational waves in 2015, the physics of tsunamis, the stress polishing of telescope mirrors and the physics of swimming all appear naturally in the development of the physics.

The authors admit that they have been strongly influenced by the magisterial tenvolume Course of Theoretical Physics - published by Lev Landau and Evgeny Lifshitz between the 1950s and the 1980s - and by The Feynman Lecture on Physics, first published in 1964. Their approach is a synthesis of these: the rigour and authority of Landau and Lifshitz, and the more intuitive arguments and flashes of insight of Richard Feynman. A plethora of hints, analogies, careful explanations and cross-references aid understanding. I particularly applaud the close attention to the realms of validity of the theoretical infrastructures that the authors create in parameter space. Also to be strongly welcomed is their inclusion of a number of topics that tend to be squeezed out of physics courses - elasticity, stress-strain relations, plasma physics and magnetohydrodynamics are a few examples.

The mathematical framework set out in Chapter 1 provides the coherence. The approach is strongly geometrically based: geometrical objects represent physical variables, what the authors refer to as coordinatefree tensor algebra. Although this is familiar to contemporary applied mathematicians and theoretical physicists, it may well be new territory for some classical-physics 
lecturers. Throughout most of the book, the mathematical essentials could be appreciated without adopting that approach, but its advantages are considerable when the authors discuss the fields in which they are international leaders general relativity, black holes and highenergy astrophysics.

All that said, I was constantly thinking about how I would have presented the same material using more elementary techniques. In my view, simpler, perhaps more intuitive, approaches can help students to appreciate the greater power of the methods adopted in this book, and would reinforce the new graduate student's capacity to master the material. For instance, Thorne and Blandford's geometrical approach leads directly to the invariant number density of states in phase space in relativity. I derive the same result more primitively, by considering the various aberration effects involved in observing a relativistically moving black body; in this way, the student appreciates more about aberration effects in relativity, as well as about a key relativistic invariant. This is not a criticism, but rather an example of the advantages of adopting multiple approaches.

The book becomes more demanding as it progresses through the subjects. The final section, on general relativity, is perhaps the most challenging, as the authors are well aware. However, their many insights are certainly worth the effort, for example in understanding the physics close to the event horizon of a rotating black hole. In the final chapter, they drop their pedagogical mantle and bring everything together in a synthesis of understanding of contemporary cosmology. This is authoritative and should be supplemented by the many excellent books on the subject - there is a vast amount of detailed physics to be mastered.

How is this book going to be used? Many separate courses could be created from it. I suspect that, as with the Feynman lectures, professors will love the approach, whereas most students will appreciate its brilliance only once they have assimilated the material through independent reading. That is what I recommend: take one of the sections and enrich it with supplementary reading (the authors make ample suggestions). Then repeat the process several times, with a progressively deeper understanding each time.

Malcolm Longair is Jacksonian Professor Emeritus of Natural Philosophy at the Cavendish Laboratory of the University of Cambridge, UK. His most recent book is Maxwell's Enduring Legacy. e-mail:msl1000@cam.ac.uk

\title{
CHEMISTRY
}

\section{Explosive moments in the laboratory}

\author{
Mark Peplow surveys a gorgeous gala of reactions in \\ Theodore Gray's new book.
}

$\mathrm{F}$ I or Theodore Gray, chemical reactions are "a sort of nanoscale fight club". In Reactions, the chemist, science writer and technologist offers a lavishly illustrated tour of this molecular battleground, full of wit and wonder.

Gray's career as a chemical evangelist began in 2002, when he misread a line in Oliver Sacks's Uncle Tungsten (Knopf, 2001) and imagined the periodic table of elements as a literal table. A skilled woodworker, Gray decided to build it and stock cavities beneath each symbol with samples of the elements.

Then, he recalls, "things really got out of hand" (go.nature.com/2fdcm9b). The table won the $2002 \mathrm{Ig}$ Nobel Prize in Chemistry, and spawned a cottage industry: Gray now sells periodic-table posters, books and quilts, and makes museum displays. With photographer Nick Mann, he has amassed a gallery of element photos, showcased in his 2009 book The Elements. Its sequel, Molecules, followed in 2014; Reactions is the final part of the trilogy (all published by Black Dog \& Leventhal).

Gray's enthusiasm shines in Reactions. Take the humble glow stick, which mixes two precursors to generate a peroxyacid ester that jolts a dye into emitting light. Of this, an object available at petrol stations for a pittance, he urges: "I insist that you be amazed." The text is peppered with dry asides, and a grumpy disdain for anything unscientific. Homeopathy he brands authorized lying; claims for 'chemical-free' health foods irk him. Even steampunk - gadgetry with a Victorian aesthetic - draws his ire, because "none of the things these people make actually work".

So far, so enticing. Yet the book struggles to sustain momentum because it lacks a narrative. In The Elements, the organizing principle was obvious: it was a beautiful catalogue of the building blocks of matter, ordered by atomic number and full of fascinating facts. But chemical reactions are

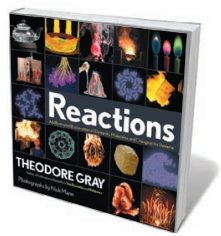

Reactions: An Illustrated Exploration of Elements, Molecules, and Change in the Universe THEODORE GRAY Black Dog \& Leventhal: 2017.

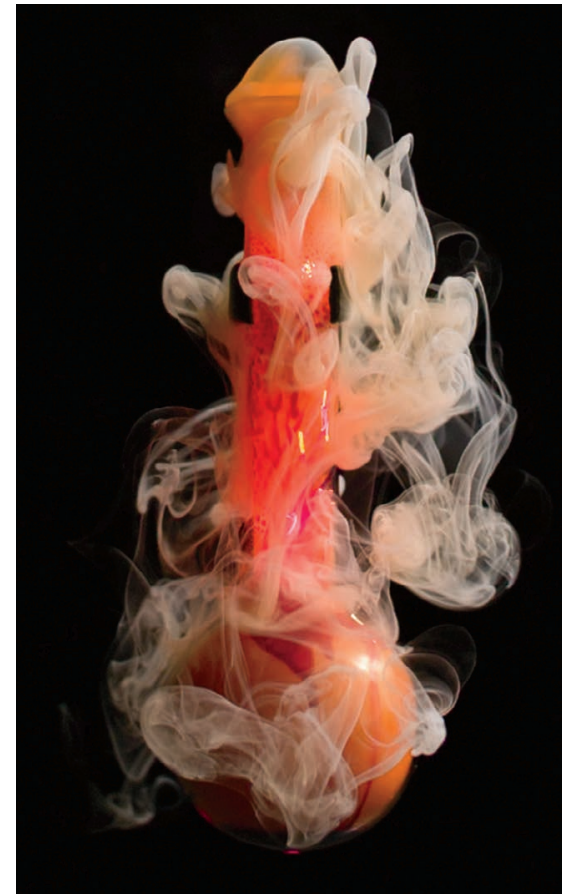

Aluminium foil reacts with bromine.

messy and multitudinous. It can be hugely challenging to explain why they occur, and to choose which to include.

Reactions leans heavily on combustion and explosion - understandable, given its visual emphasis. As a result, some of it feels samey. Plenty of other glamorous reactions could have illustrated different concepts, from the redox chemistry behind the spectacular ammonium dichromate 'volcano', to the catalytic decomposition of hydrogen peroxide used to create 'elephant's toothpaste' - a foam fountain beloved of science demonstrators.

When Gray settles on a subject, he can provide genuine insight, as in sections on the anatomy of fireworks or the composition of paints. But too often, the coverage is frustratingly superficial. In the sole example from the vast field of synthetic organic chemistry, he outlines the total synthesis of the alkaloid physostigmine, yet divulges nothing about why it is useful - it's a treatment for glaucoma. And although the diagrams that show its step-by-step construction create an impression of complexity, Gray doesn't begin to explain why this particular route is a 